\title{
Resiniferatoxin for Pain Treatment: An Interventional Approach to Personalized Pain Medicine
}

\author{
Michael J. Iadarola*,1,2 and Gian Luigi Gonnella ${ }^{1,3}$
}

${ }^{I}$ Neurobiology and Pain Therapeutics Section, Laboratory of Sensory Biology, National Institute of Dental and
Craniofacial Research, NIH; ${ }^{2}$ Building 49 Room 1C20, 49 Convent Drive MSC 4410, Bethesda MD 20892-4410, USA;
${ }^{3}$ Department of Anesthesiology and Intensive Care Medicine, Catholic University School of Medicine, Rome, Italy

Abstract: This review examines existing preclinical and clinical studies related to resiniferatoxin (RTX) and its potential uses in pain treatment. Like capsaicin, RTX is a vanilloid receptor (TRPV1) agonist, only more potent. This increased potency confers both quantitative and qualitative advantages in terms of drug action on the TRPV1 containing nerve terminal, which result in an increased efficacy and a long duration of action. RTX can be delivered by a central route of administration through injection into the subarachnoid space around the lumbosacral spinal cord. It can also be administered peripherally into a region of skin or deep tissue where primary afferents nerves terminate, or directly into a nerve trunk or a dorsal root ganglion. The central route is currently being evaluated as a treatment for intractable pain in patients with advanced cancer. Peripheral administration offers the possibility to treat a wide diversity of pain problems because of the ability to bring the treatment to the site of the pain (the peripheral generator). While not all pain disorders are appropriate for RTX, tailoring treatment to an individual patient's needs via a selective and local intervention that chemically targets a specific population of nerve terminals provides a new capability for pain therapy and a simplified and effective approach to personalized pain medicine.

Keywords: Calcium cytotoxicity, osteosarcoma, osteoarthritis, dog, cancer pain, geriatric, ion channel, malignant pain, nonmalignant pain, spinal stenosis, arachnoiditis, complex regional pain syndrome, neuropathic pain, C-fibers, A-delta fibers, CGRP, Substance P, mitochondria, endoplasmic reticulum, plasma membrane.

\section{SCOPE OF REVIEW}

This review will introduce some of the background and unique features of using resiniferatoxin as a pain control agent. We compare RTX to different analgesic agents currently available and also examine the types of human pain problems that RTX might or might not be suitable for, and what some of the criteria are for such an assignment. We examine the differences between a vanilloid antagonist and a vanilloid agonist for pain control. For RTX two main routes of administration are distinguished, intrathecal and peripheral, each has its advantages and clinical indications for use. Several tables are used to summarize these points. The review also examines some of the existing preclinical animal and early clinical results with RTX, again, critiquing what they show, how RTX performed and what advantages might accrue with its use. The review is grouped around proposed clinical uses of RTX and the necessary routes of administration. A few possibilities for directions forward that can shape a personalized approach to pain control are discussed in closing.

\section{INTRODUCTION}

The sheer diversity of pain disorders and the multiplicity of locations in the body in which pain can occur, literally

\footnotetext{
*Address corresspondence to this auhtor at the Building 49 Room 1C20, 49 Convent Drive MSC 4410, Bethesda MD 20892-4410; Tel: 301-496-2758; Fax: 301-402-0667; E-mail: miadarola@dir.nidcr.nih.gov
}

from the head (facial nerve injury) to the toe (Morton's neuroma) presents, if not a bewildering, then certainly a complex array of possibilities for pathological pain generating mechanisms and for treatments. This multiplicity and complexity makes identifying unifying principles, critical mechanisms and molecular targets for therapeutic intervention challenging propositions. The conceptual pendulum for pain treatment can swing towards favoring central nervous system mechanisms to the opposite pole of peripheral nociceptive neurons. The main elements considered in this review are as follows: the peripheral nervous system can be targeted by RTX to produce analgesia, the local administration of RTX further enhances specificity and reduces potential side effects and local injection can be adapted to treat many different types of pain problems. This is the essence of the idea of an interventional approach to personalized pain medicine.

The central and peripheral nervous systems each have their advantages and limitations for analgesic manipulations. Molecular targets located in both sites have been the subjects of intensive analgesic drug development efforts, with peripheral targets receiving the most attention over the past 30 years. Several pain generating mechanisms and molecular targets and treatments are summarized in Tables $\mathbf{1 A}$ and $\mathbf{B}$, respectively. While the list is not exhaustive, it serves to highlight the apparent diversity of pain mechanisms. Nonetheless, the pain problems listed in the table all have one thing in common: activation of the peripheral nerve. 
Selective interference with the peripheral nerve is where an axonal- and/or nerve terminal-directed agent like RTX can exert analgesic activity against many types of pain, despite different locations and origins.

Table 1. (A) Sampling of peripheral mechanisms contributing to generation of nociceptive signals and (B) current or potential therapeutic targets

\begin{tabular}{|l|}
\hline A) Mechanisms Contributing to Persistent Nociceptive Signaling. \\
\hline \hline Post-injury tissue remodeling (scars, neuromas, adhesions) \\
\hline $\begin{array}{l}\text { Pressure or entrapment of a peripheral nerve (lumbar disk herniation, } \\
\text { carpal tunnel syndrome) }\end{array}$ \\
\hline Nerve injuries and demyelination leading to hyperexcitiability \\
\hline Chronic Inflammatory conditions \\
\hline $\begin{array}{l}\text { Compromised blood supply and ischemia (sickle cell disease, vascular } \\
\text { claudication/obstructive arteriopathy) }\end{array}$ \\
\hline $\begin{array}{l}\text { Infectious diseases or post-infectious mechanisms (shingles, post- } \\
\text { herpetic neuralgia) }\end{array}$ \\
\hline B) Current or Potential Peripheral Therapeutic Targets \\
\hline $\begin{array}{l}\text { Ion channel mechanisms underlying repetitive firing of nociceptors } \\
\text { (pregabalin, gabapentin) }\end{array}$ \\
\hline Blockade of calcium ion channel (ziconitide) \\
\hline $\begin{array}{l}\text { Blockade of sodium ion channel (lidocaine and SNS/TTX resistant Na } \\
\text { channels). }\end{array}$ \\
\hline Activation of K channels (retigabine) \\
\hline $\begin{array}{l}\text { Block of algesic receptors on afferent nerve endings (e.g., TRPV1, } \\
\text { TRPA1, bradykinin, prostaglandin, ATP receptors, etc.) }\end{array}$ \\
\hline $\begin{array}{l}\text { Receptors mediating presynaptic activity of primary afferent endings } \\
\text { (Mu opioid receptor) }\end{array}$ \\
\hline $\begin{array}{l}\text { Selective destruction of nociceptive nerve endings (e.g., with capsaicin } \\
\text { or RTX) }\end{array}$ \\
\hline A \\
\hline
\end{tabular}

\section{BACKGROUND}

Prior to the successful cloning of the TRPV1 channel, many efforts were focused on developing antagonists against receptors for substance $\mathrm{P}$ [1], bradykinin [2], COX 2 inhibitors [3], and capsaicin [4]. Historically, it is interesting to consider Substance P. The enrichment of substance P in the spinal cord dorsal horn generated much interest and findings from these investigations formed a conceptual basis for the development of neurokinin 1 receptor antagonist analgesic drugs [5]. The start of this effort antedated the full understanding of the transmitter complexity of single neurons [6-8], the fact that the primary afferents contain a cornucopia of peptides and, importantly, the excitatory amino acid glutamate [9]. A more complete appreciation of the multifactorial neurochemical nature of nociceptive transmission may have tempered expectations of the efficacy of blocking just one neuropeptide transmitter in the $\mathrm{C}$-fiber repertoire [10].

The obvious main advantage of peripheral targets is the reduced potential for CNS side effects. For example, nausea and sedation are prominent clinical manifestations that can accompany opioid analgesia [11]. The list of potential peripheral targets received a large supplementation subsequent to the cloning of the capsaicin receptor [12] called TRPV1 (termed VR1 at the time) and other thermoand chemoresponsive channels from DRG [13-15]. TRPV1 denotes the transient receptor potential channel family $\mathrm{V}$ number 1 and is a member of a large super-family of TRP channels first identified in Drosophila $[16,17]$. The further identification of multiple thermo- and chemo-responsive TRP channels in DRG neurons launched a resurgence in the development of potential analgesic drugs that could be antagonists of the various TRP channel members $[15,18]$. Other, specific targets are the tetrodotoxin-insensitive, sensory neuron-specific (SNS) sodium channels [19], first identified by subtraction cloning of DRG transcripts [20] and the TrkA receptor, which can be antagonized by using antibodies to its cognate ligand nerve growth factor (NGF) to block NGF-mediated nociceptor sensitization [21]. Additional peripherally directed candidate mechanisms may include not only ion channels and GPCR's but also molecules that engage in nerve regeneration and repair mechanisms to augment or accelerate the healing process for damaged nerves [22-24]. In many cases, drug development efforts and human testing for these approaches, such as the anti-NGF antibodies, have reached very advanced stages, but clinical trials and safety evaluations are ongoing $[25,26]$.

Systemic versus local: One element that most of the aforementioned treatments have in common is that they are administered systemically, either orally or by injection (e.g., anti-NGF antibodies). The result is that the entire body is exposed to the drug, which can increase the potential for off target actions or actions on the intended molecular target when it is expressed in multiple tissues. Most of the current antagonists of the orthosteric capsaicin binding site on TRPV1 block the ability to sense painful heat throughout the body, thereby leaving a patient vulnerable to damaging thermal stimuli. After treatment with an antagonist, hot temperatures are perceived as warm or innocuous [27], and potentially increasing the risk of a burn injury. This is an important consideration in the course of activities of daily living (ADL). The orthosteric antagonists also have a tendency to increase core body temperature [28, 29] and impact other elements related to diagnostic signs and symptoms associated with disease states. This is a subject previously discussed in the context of a "perfect analgesic" in two short communications [30, 31].

A relatively simple way to avoid non-intended actions and/or global effects resulting from systemic administration is to deliver a drug locally. However, very few pain treatment approaches have the necessary pharmacodynamic and pharmacokinetic characteristics to make this practical. For example, local anesthetics are phenomenally useful drugs, but their short duration of action and broad-spectrum blockade of peripheral nerve fiber types make them unsuitable for long-term treatment of chronic pain problems. Feelings of numbness, complete insensitivity to mechanical, thermal, chemical and inflammatory pain and the loss of muscular strength and proprioception occuring at higher doses may also complicate effective, long-term implementation [32]. Local anesthetics can also cause toxicity with prolonged administration at some sites such as the cornea [33]. This example provides two important 
Table $2 \mathrm{~A}$ and $\mathrm{B}$. Differences between local peripheral and intrathecal administration of RTX and comparison to systemic TRPV1 antagonists

\begin{tabular}{|c|c|c|}
\hline \multicolumn{2}{|c|}{ A. Peripheral } \\
\hline \hline Effect or property & $\begin{array}{c}\text { Vanilloid } \\
\text { Antagonist }\end{array}$ & $\begin{array}{c}\text { RTX } \\
\text { (Vanilloid Agonist) }\end{array}$ \\
\hline Integrity of nerve terminal & Intact & Days to weeks \\
\hline Duration of action & Hours & Nerve ending dies back secondary to calcium overload \\
\hline Route(s) of administration & Oral & High \\
\hline Selectivity for TRPV1 receptor & High & $\begin{array}{c}\text { Lost due to calcium overload and nerve terminal } \\
\text { inactivation }\end{array}$ \\
\hline Capacity for response to other algesic substances & Possible & Yes, when nerve ending regenerates \\
\hline Reversibility & $\begin{array}{c}\text { Yes, based on pharmacokinetic } \\
\text { profile }\end{array}$ & Site of injection \\
\hline Coverage & Entire body & \\
\hline
\end{tabular}

\begin{tabular}{|c|c|}
\hline \multicolumn{2}{|c|}{ B. Intrathecal RTX } \\
\hline Capacity for response to other algesic substances & $\begin{array}{l}\text { Lost due to calcium overload and dorsal root ganglion neuronal loss or } \\
\text { axotomy }\end{array}$ \\
\hline Coverage & $\begin{array}{l}\text { Dorsal roots and ganglia; effect varies with volume and dose of injection. If } \\
\text { given into the lumbar cistern then the cauda equina and lumbo-sacral DRG } \\
\text { are exposed. }\end{array}$ \\
\hline
\end{tabular}

considerations for effective analgesia when an analgesic agent is applied by regional injection or infiltration: one is duration of action and the other is fiber type selectivity.

\section{Retention or Loss of Pain Modalities After RTX in Chronic and Acute Pain Conditions}

A subtext of selectivity is the retention of some nociceptive sensitivity: complete loss of pain sensitivity can be life threatening. Even the complete loss of one modality (e.g. hot thermal pain) is not desirable for long term care because it can negatively affect ADL [34]. One advantage of RTX in comparison to local anesthetics is that it is selective for the nociceptive population of primary afferent fibers and, indeed, a subpopulation of nociceptive afferents [35-37]. The sensations of mechanical pinch and pressure are largely intact following RTX administration, as are sensations of vibration and cold temperature. Furthermore, proprioceptive sensations necessary for locomotion are unaffected. Rats injected with RTX intrathecally can walk on a Rotorod in a similar fashion as vehicle injected rats and for a similar duration [36]. We also see no motor impairment in dogs injected intrathecally with RTX by either the intracisternal or lumbar puncture routes [37]. Data from multiple studies support the conclusion that the selectivity afforded by RTX spares motor axons and other sensory inputs, which is a significant factor when assessing safety, ADL, and quality of life. These are important considerations for the intrathecal route because it affects many dorsal roots at once [36], but they are equally important when considering local administration paradigms. For example, the sparing of mechanoreceptors is critically important for corneal applications, where the blink reflex must remain intact, [38] Similarly for joint injections, feedback from specialized muscle spindle and Golgi tendon organ proprioceptors is an essential component of muscular coordination. Sparing of mechano-responsive nociceptive axons and nerve endings may also help protect against damage to the joint from inappropriate use. All of these considerations obviously apply to chronic pain conditions where long-term analgesia and side effects are important elements.

RTX can also be used for acute conditions where nerve terminals are damaged or will be damaged (e.g., a proposed elective surgery). Two potential examples are the use of RTX to control burn or post-operative pain, respectively. In the former case RTX application would occur after the injury. In the latter case RTX would be applied in a preemptive fashion. In both cases the nerve terminals in the pain zone are the targets, although the formulation of the drug and the means of administration may be different. In summary, pain treatment with RTX can encompass a wide variety of acute and chronic pain problems with two provisos: firstly, the drug must contact the TRPV1 molecule as it resides in the nerve terminals, axons or neuronal cell bodies of TRPV1-expessing sensory ganglionic neurons. Secondly, the injection site must coincide with the neurons causing the pain. Thus issues of etiology, pain localization, accessibility to injection, duration of exposure to RTX and spread of the drug from the site of injection are all aspects of a personalized interventional approach that will govern optimal therapeutic outcome.

\section{Differences Between Peripheral and Intrathecal Routes of Administration}

The above introduction suggests that RTX can be a versatile agent for treatment of a wide variety of pain problems. Two main routes of administration, peripheral and intrathecal, provide an appropriate framework for conceptualizing how to use this compound and are summarized in Table $\mathbf{2 A}$ and $\mathbf{B}$. The main feature of the 
intrathecal (and intraganglionic) route of administration is a permanent loss of connectivity between TRPV1-expressing neurons and the spinal cord. This can arise either by loss of the neuronal cell body in the DRG or loss of the TRPV1 axons in the dorsal root. For both intrathecal and intraganglionic routes the effect is permanent: neither the neuron nor its centrally projecting axon regenerate [36]. This contrasts with the effects of peripheral administration. Studies in animals show that the peripheral sensitivity returns to a level that is not significantly different from baseline subsequent to subcutaneous, perineural or topical administration [35, 38-40]. We also compare TRPV1 antagonists in Table $\mathbf{2 A}$.

Here again, there are major mechanistic differences that serve as relevant guides to clinical use. First, when used effectively, TRPV1 agonists will literally sever the connection between the body and the spinal cord for the TRPV1-expressing subpopulation of afferents. When given peripherally this can occur by calcium overload of the peripheral terminals [41-44] or, when given intrathecally, loss of the neuronal cell body or its centrally projecting axons [35-37]. Peripherally, the loss of the nerve ending renders the afferent nerve insensitive to all of the different receptors for algesic substances that it can react to. The net result is a broader spectrum of "analgesia" than might be obtained with a TRPV1 orthosteric capsaicin site antagonist. Second, duration of action is another difference (discussed in more detail below) but usually the effect of peripheral nerve terminal inactivation is on the order of several days, weeks or months depending on the injection site. Third, as mentioned previously, the volume of distribution is vastly different. A systemic antagonist will affect the entire body, whereas peripherally applied RTX is site specific, intraganglionic application is dermatome specific, and intrathecally applied RTX can affect multiple dermatomes, mainly in the lower half of the body when given into the lumbar cistern.

\section{Actions after Topical Administration}

After peripheral administration, the speed with which function is restored depends on several factors. First, and most important is the proximity of the nerve ending to the site of RTX administration. Second, the location of testing in relation to the site of administration is also crucial. An allied question is how much regeneration is needed to restore function? In our experience with the cornea [38], nocifensive function, assessed by eye wipe response to corneal application of capsaicin, returns within a matter of days $(\sim 4)$. The return of this behavioral response coincides with the reinnervation of the cornea by CGRP containing afferent endings. Immunocytochemical staining showed that regeneration of only a fraction of the original number of nerve endings was sufficient to restore the eyewipe response. In this study 10 microscopic fields in each cornea, double labeled for beta-tubulin and CGRP were examined at 40X, with the condition that a field had to include beta-tubulin to be counted. In control corneas, $94 \%$ of beta-tubulin fields were positive for both tubulin and CGRP. By 24 hours postRTX administration, only $18 \%$ of the fields were positive for CGRP. By 12 days, the fibers had largely returned but the process was not $100 \%$ complete. Nonetheless, sensitivity recommenced by 5 days. Thus, enough TRPV1-containing nerve terminals regenerate to the corneal surface to restore full behavioral function at a $0.1 \mu \mathrm{g} / \mu \mathrm{l}$ dose and nearly full noci-responsiveness following a $1 \mu \mathrm{g} / \mu \mathrm{l}$ dose. The methods used were quite straightforward and the data clearly demonstrated the temporary effect of local, topical, peripheral administration of RTX.

One of the main points to extract is that nociceptive function can return before full axonal re-innervation has occurred. This supports the idea that measurement of both parameters is informative for assessment of the full spectrum of agonist actions and for the interpretation of studies conducted at various sites in the body. The analgesic duration of locally applied RTX may be quite different at different sites in the body. Indeed, the cornea may be somewhat unique in terms of the rapidity of functional reinnervation compared to the other routes of administration. It is also possible that this effect may be influenced by the test itself (capsaicin eye wipe) versus the usual paw thermal tests. Other peripheral routes of RTX administration can exhibit a more prolonged effect (e.g. subcutaneous or perineural) or a more widespread effect (e.g. intraperitoneal) [45-47] and whether the cell body and/or central axon is affected (intrathecal/intraganglionic). Additionally, the interval needed for full re-innervation will be influenced by the relative density of innervation of TRPV1-containing Cand A-delta fibers [48].

Topical cutaneous application of RTX to the skin is not discussed here. Our unpublished data, using various formulations of RTX and a wide range of concentrations failed to elicit nocifensive behaviors in rats when applied to the dorsal and plantar surfaces of the hind paw. We interpreted the lack of behavioral responses as evidence that RTX did not cross the skin very efficiently. In fact, the doses needed for the eye are quite high (100 nanograms $/ \mu 1)$ compared to those needed to elicit nocifensive behaviors following subcutaneous injections of RTX (0.5 nanograms $/ \mu 1)[38,49]$. However, topical capsaicin is used clinically and the $8 \%$ capsaicin patch can produce therapeutically significant effects for up to 12 weeks [50], which is consistent with RTX and capsaicin preclinical actions $[35,51]$. The similarity between the two agonists in terms of duration suggests that, once the nerve endings undergo axonopathy, the steps necessary for repair and the time required are similar.

In the next several sections the effects of two other routes of peripheral administration, subcutaneous and perineural, will be discussed with emphasis on differential actions, effect of dose and duration of drug effect and advantages of the different routes and their potential uses.

\section{Actions after Subcutaneous Administration, C- and A- delta fibers}

Effects obtained with other routes of administration do not necessarily follow the rapidity of return of nociceptive responsiveness obtained in the cornea. Early studies of subcutaneous RTX administration show that both the degree and duration of action of RTX, injected into the footpad, were dose-dependent [35]. Duration applies to the acute nocifensive actions of RTX that occur within 3 to $7 \mathrm{~min}$ upon injections [49] and to the duration of subsequent analgesic actions. The duration of acute nocifensive activity 
was inversely related to the dose: low doses produced a more prolonged effect than higher doses. For example, a dose of $50 \mathrm{ng}$ in $100 \mu \mathrm{l}$ produced paw shaking and licking behaviors that lasted more than $70 \mathrm{~min}$. Compare this to a $100 \mathrm{ng}$ in $100 \mu 1$ dose, for which nocifensive activity lasted approximately $30 \mathrm{~min}$ [49] and a higher dose of $625 \mathrm{ng}$, where nocifensive activity lasted less than $10 \mathrm{~min}$ [35]. In summary, the dose-related rapidity of nerve terminal inactivation is consistent with a dose-related increase in nerve terminal calcium cytotoxicity. The higher the dose, the faster the transition to the inactivated state. Similarly, the duration of post-injection analgesic activity is also directly related to dose: the higher the dose, the longer and more profound the duration of local analgesic activity. This can last for 1 to 5 weeks depending on the stimulus intensity and fiber type stimulated [48].

\section{A-delta Versus C-fibers}

Earlier studies [35, 36] used a Hargreaves device or hot plate [39] to assess thermo-sensitive primary afferents and thermal hyperalgesia. While the device we used up until $2010[48,52,53]$ was the forerunner of the commercially available device, both the commercial device and the "beta versions" depend on a white light radiant heat source and neither distinguishes between $\mathrm{C}$ - and A-delta fibers. In more recent investigations, we now use an infrared diode laser to differentially activate A-delta and C-fiber thermonociceptors. These experiments showed that subpopulations of both fiber types detect noxious thermal stimuli via TRPV1 and that both are susceptible to local axonopathy produced by injection of RTX. We also demonstrated that the apparent time for full recovery of the A-delta population was longer than that for the C-fiber population [48]. The difference was attributed to the fact that the A-delta fibers, because of the myelination and the presence of nodes of Ranvier, are structurally more complex than the C-fibers. Therefore, reconstructing breakage at a node of Ranvier in an A-delta fiber might be more time consuming. If breakage occurs at multiple nodes then reconstruction might be even more time consuming for the A-delta fibers than the C-fiber population.

\section{Implications of Topical and Subcutaneous Administration}

\section{Developmental Aspects}

RTX can be administered into several body compartments (e.g. subcutaneous or into a joint) and it is of interest to examine the potential impact that primary afferent developmental biology might have upon the actions of subcutaneous or deep injections of RTX. Recent studies show that several neuronal lineages differentiate during development to yield the multiple modalities of nociception and somatosensation that we experience [54] (e.g. touch pressure, pinch, itch, cool, cold, warm, hot, vibration, hair movement, etc.) $[24,55-57]$. Deep and superficial sites in the body receive a differential innervation in terms of developmental lineage. Different subsets of neurons innervate different cutaneous specialized nerve endings or types of hairs and influence pain sensation [58-60]. In mice the CGRP-containing peptidergic and Mrgd receptor neurons terminate at differential depths in the epidermis [61] and transduce two distinct modalities: heat pain and mechanical pain, respectively. Epidermal innervation by the TrkA lineage neurons appears to be greatly reduced upon conditional knockout of Runx1 transcription factor in sensory neurons (Ma, Q, personal communication). It is possible such developmental specification will apply to anatomical and functional specification of DRG innervation of deep tissue such as muscle and joints [59]. For example, it has been reported that the isolectin $\mathrm{B} 4$ positive $\left(\mathrm{IB} 4^{+}\right)$ population of non-peptidgergic neurons does not innervate the rat knee joint [62]. Thus, factors specifying the fiber type(s) that innervates a particular site can play an important role in guiding the mechanistic-based usage of RTX.

\section{Therapeutic Implications}

Certain practical conclusions for therapeutic implementation of RTX can be drawn from the results of subcutaneous and topical administration studies for pain control. For example, based on dose differentials for topical versus subcutaneous administration, it seems likely that superior pain control would be obtained for post-surgical incisional pain by delivering RTX through a series of subcutaneous injections along the line of the incision compared to topical application. Even if the compound was applied to the wound margins after the incision was made, penetration to the nerve terminals is likely to be diminished by dilution along the exposed wound edges. The clinical experience with capsaicin is informative in this regard. Subsequent to a pre-operative block with lidocaine, the incision site for bunionectomy was superfused intraoperatively with $1000 \mu \mathrm{g}$ of capsaicin in $4 \mathrm{ml}$ of vehicle immediately before wound closure. This produced a reduction in post-operative opioid use and a significant reduction in mean visual analog scale rating of pain (a decrease of 12.7 and $14.2 \mathrm{~mm}$ ) at 8 and 24 hours postoperation, a phenomenon not seen during the remaining two to 14 days of the study [63]. A larger study of hernia repair also using $1,000 \mu \mathrm{g}$ of capsaicin showed significant analgesia from incisional infiltration with capsaicin [64]. It is interesting to note that this amount of capsaicin clearly did not produce a thorough nerve terminal inactivation at the operative site. We attribute this mainly to dilution of the drug at the site and the pharmacodynamics of channel activation: capsaicin allows the channel to open and close whereas RTX causes a prolonged channel opening [65]. Thus, capsaicin is very effective at stimulating TRPV1 but less effective than RTX at inactivating the TRPV1containing nerve terminal. The timing of drug administration, which was after the surgery as opposed to before (preemptively), may also have contributed to reducing efficacy. These considerations emphasize how important procedural factors are in determining the effectiveness of a local interventional approach.

\section{Dose-response}

The observation of an inverted dose-response is another useful parameter to explore for clinical dose estimation and prediction of effects. With RTX, a very low dose provides a remarkable amount of stimulation prior to nerve terminal inactivation and therapeutic benefit. This excess stimulation can add to the central sensitization that would result from the surgical procedure alone and may be counterproductive for an analgesic action (as suggested above for capsaicin). Thus, a minimally effective dose for inactivation likely needs to be 
higher than that for TRPV1 stimulation only. The inverse dose-effect relationship between stimulation and inactivation needs to be taken into consideration when using RTX therapeutically.

\section{Body Compartments}

Lastly, the characteristics of the body compartment may play a role. If the site of injection provides for rapid diffusion then the peak effect of RTX may be reduced. Conversely, if the compartment confines the drug then the analgesic effect may be more pronounced. The observed efficacy of capsaicin injections into knee joints in osteoarthritis appears to support this idea. Four out of five osteoarthritis patients injected intra-articularly with $1,000 \mu \mathrm{g}$ of capsaicin exhibited reduced pain for a period of time between two and five weeks [66]. However, increased efficacy due to confinement of the agonist to a body compartment does not appear to be universally advantageous since instillation of RTX into the bladder has had a very mixed effect on interstitial cystitis [67-70].

\section{Actions after Perineural Administration}

In general, when a local anesthetic is applied to a peripheral nerve, it cannot produce analgesia without at least some effect on non-pain-related motor and sensory functions. So the resulting condition from a nerve block through local anesthetic administration is named "conduction anesthesia" [71]. Unlike the local anesthetics, RTX elicits a phenomenon termed "conduction analgesia", where analgesia can be produced without having any effect on motor and sensory modalities unrelated to nociception [72]. The rationale for perineural administration is based on the action of RTX on nociceptive fibers in the nerve trunk, mediated by its interaction with TRPV1 receptors residing in the axon. TRPV1 is found in all parts of the primary afferent neuron, from peripheral terminals to central endings in the spinal cord $[73,74]$. Calcium imaging of DRG neurons in primary culture demonstrated that RTX can produce direct calcium cytotoxicity on the axon as well as the neuronal perikarya [75].

\section{Effects on Experimental Pain Models}

Given these neurobiological and pharmacological underpinnings, perineural RTX administration was shown to prevent the development hyperalgesia using the Bennett mononeuropathy model [40]. A single percutaneous application of $0.5 \mu \mathrm{g}$ RTX in the vicinity of the sciatic nerve, three hours before the placement of loose constrictive ligatures around the nerve, prevented the full expression of heat and mechanical hyperalgesia. In another study Neubert et al. evaluated low, graded doses of perineural RTX as a method for regional pain control. They observed a significant inhibition of thermal and mechanical nociception, in particular, heat hyperalgesia that was dose- and timedependent. Perineural RTX administration did not affect normal proprioception or motor control as tested by rotorod performance at 1 day and 1 week post-injection. Other pain sensations and mechanical detection thresholds were preserved and the analgesic behavioral actions were reversible over a two-week period. Thus, the action of RTX on peripheral nerve displays the same selectivity, in terms of fiber types affected and spared, as is seen in the DRG after intraganglionic administration: only the nerves that contain TRPV1 are affected and there is no bystander effect on nonTRPV1 expressing axons or perikarya. In fact, using electron microscopy, it was difficult to detect any change at all in the sciatic nerve after perineural RTX administration [40]. The expectation was that some modification of the nerve would be visible and several explanations were offered as to the lack of directly observable impact. In contrast, in vitro studies show a profound effect of RTX on primary DRG neuronal cell bodies and processes [41, 75] and, in the cornea, activation of calcium transients in nerve endings [43, $44,76]$ and an apparent lesion of the axon as determined by loss of CGRP staining [38]. However, loss of CGRP staining, while consistent with the idea of an axonal lesion, does not directly demonstrate an actual lesion. Thus, the exact process of nerve inactivation requires further investigation.

\section{Therapeutic Considerations}

Peripheral nerves are obvious targets for a drug that has the capability of interacting with axons. As noted above, fiber type specificity and pain modality are of paramount importance. However, when pain is intractable even nonspecific neurosurgical interventions are used such as cutting the peripheral nerve (neurectomy). While neurectomy can provide pain relief, it can also instate feelings of numbness and lead to the formation of a painful neuroma at the cut nerve stump. The advantages of perineural RTX administration are fiber and modality selectivity, a long duration of action and, obviously, anatomical specificity. One potential disadvantage is the potential for an incomplete distribution of the drug among the fascicles of a large peripheral nerve. Imaging of percutaneous sciatic perineural injection of fluorescein isothiocyanate showed that some nerve bundles were more brightly fluorescent than others. This is consistent with the idea that, while the needle tip can be demonstrated to be near the nerve bundle with electrical stimulation, the concentration gradient of drug affects the part of the nerve nearest the needle tip most efficiently (Neubert 2008, figure 1). For large nerves there is an even greater propensity for heterogenous distribution. In one experiment, a horse with chronic pain in the hoof was treated. The relevant nerve was quite large so we chose to expose the nerve and inject directly into it rather than use a percutaneous perineural application. This produced an evident, but transient, analgesic effect that allowed the horse to be ridden for about 4 months after injection (Iadarola, unpublished). The main procedural advantages here are that perineural or direct intra-nerve injection approaches can be tailored to an individual's presentation of their pain problem. For example, if an injury involves more than one nerve, then an optimal injection procedure can be designed that will target each of the nerves or branches involved.

Table 3 outlines some of the pain problems that might be ameliorated by localized treatment with RTX, and highlights the inherent flexibility of a procedure-based approach for personalized pain management.

\section{RTX: Intraganglionic Administration}

Neurons of the dorsal root and trigeminal ganglia receive noxious and somatosensory information from defined anatomic areas of the body called dermatotomes. In a sense 
Table 3. Pain conditions that may be susceptible to treatment with local injection or topical Resiniferatoxin

\begin{tabular}{|c|c|c|c|}
\hline Condition & Location & Current treatment(s) & RTX treatment \\
\hline Morton's Neuroma & Foot, between $3^{\text {rd }}$ and $4^{\text {th }}$ toes & $\begin{array}{c}\text { Steroid injection, Cryogenic neuroablation, } \\
\text { Decompression surgery, Removal of the } \\
\text { neuroma }\end{array}$ & Direct injection into the neuroma \\
\hline Localized nerve injuries & Various locations & Gabapentin pregabalin, Antidepressants & $\begin{array}{l}\text { Local infiltration of the trigger } \\
\text { zone if identifiable }\end{array}$ \\
\hline Burns & Site of burn injury & $\begin{array}{l}\text { Opioids, NSAIDs, acetaminophen, local } \\
\text { anesthetics, anxiolitics }\end{array}$ & $\begin{array}{l}\text { Topical to burn site or direct or } \\
\text { perineural injections }\end{array}$ \\
\hline Complex Regional Pain syndrome & Various locations & $\begin{array}{l}\text { Gabapentin pregabalin } \\
\text { Antidepressants }\end{array}$ & $\begin{array}{l}\text { Direct injection infiltration into } \\
\text { trigger zone if identifiable }\end{array}$ \\
\hline Osteoarthritis & Affected joints & NSAIDs or acetaminophen, opioids & Direct injection into joint \\
\hline Post-incisional pain & Site of surgical incision & $\begin{array}{l}\text { NSAIDs, acetaminophen, opioids, local } \\
\text { anesthetic instillation }\end{array}$ & $\begin{array}{l}\text { Direct injection into wound } \\
\text { margins, preemptive }\end{array}$ \\
\hline Low back pain & Affected Lumbar vertebrae & $\begin{array}{l}\text { NSAIDs, acetaminophen, opioids, local } \\
\text { anesthetic instillation } \\
\text { RF facet joint treatments } \\
\text { Surgery }\end{array}$ & $\begin{array}{l}\text { Direct injection into lumbar } \\
\text { nerve root(s), } \\
\text { Infiltration of facet joint }\end{array}$ \\
\hline $\begin{array}{l}\text { Chronic Gynecological Pain } \\
\text { (vulvodynia) }\end{array}$ & Vaginal vestibule & $\begin{array}{c}\text { Medications as above } \\
\text { Surgical tissue removal in some cases }\end{array}$ & Direct injection into trigger zone \\
\hline
\end{tabular}

targeting the ganglion can be considered a variation on injecting peripheral nerves. There is one major difference: the intraganglionic route has a high probability of being permanent. Once the neuronal cell bodies are exposed to RTX, calcium cytotoxicity will occur in the neuronal perikarya $[40,41]$ rather than a spatially remote nerve ending in the skin or a joint. If the calcium influx is sustained and strong enough cell death may result in a matter of minutes [40]. Both functional studies using live cell imaging or histological analysis after intraganglionic injection show that the toxicity is confined to cells or neurons that highly express TRPV1 [36, 40, 41, 71, 75].

RTX has been injected unilaterally into the trigeminal ganglia of rodents and monkeys. In both species, intratrigeminal RTX injection produced a unilateral block of the eye-wiping response evoked by intraocular capsaicin drops $[40,77]$. The blockage of the eye-wiping response had a rapid onset (the first test was $24 \mathrm{~h}$ after the microinjection). In the rat, the effect was essentially permanent: the capsaicin eye-wipe response was blocked for 350 days (the time of the last test). In the monkey, full blockage was present at the last test performed at 4 months. Perineural injections block neurogenic inflammation in the hind paw regions innervated by the sciatic nerve [78]. Similarly, intratrigeminal RTX blocked neurogenic inflammation specifically over the trigeminal dermatomes. This was dramatically demonstrated by Evans Blue staining in both rat and monkey. The noninjected half of the face was blue, due to extravasation of blue-stained albumin, and the side injected with RTX remained white because the afferent endings were eliminated $[40,77]$. Nociceptive behavioral responses to chemical or high-thermal stimulation and neurogenic inflammation were blocked, but at the same time low threshold mechanosensation, corneal responses to touch and liquids, and facial motor functions remained intact.

\section{Effect on Neuropathic Pain}

Intratrigeminal or close nerve root injections also block experimental neuropathic pain. Rat lumbar dorsal root ganglia (L3-L6) were injected with RTX before and after a photochemical sciatic nerve injury (Tender GC et al., 2008). The preemptive administration of RTX blocked development of tactile allodynia. RTX treatment also elevated the tactile threshold for withdrawal in rats with an established neuropathic pain condition. Taken together, these data suggest that intraganglionc RTX would be effective against a broad range of inflammatory and neuropathic pain conditions. A preemptive therapeutic effect in a loose ligature model was also seen with perineural RTX administration [79].

\section{Therapeutic Considerations}

These data support the idea that intraganglionic RTX is effective, selective, and safe. Obviously, the quality of the injection technique will be a determinant of the outcome. To assist positioning of the injection needle, various image- 
Table 4. Non-malignant chronic pain conditions that may be treated with intrathecal or intraganglionic resiniferatoxin

\begin{tabular}{|c|c|c|c|}
\hline Condition & Location & Current Treatments & RTX administration \\
\hline \hline Post-herpetic Neuralgia & $\begin{array}{c}\text { Various dermatomes, } \\
\text { frequently on the torso }\end{array}$ & $\begin{array}{c}\text { Tricyclic antidepressants, } \\
\text { Capsaicin topical, } \\
\text { Corticosteroids, Antiviral agents, } \\
\text { Lidocaine patch, Anticonvulsants }\end{array}$ & $\begin{array}{c}\text { Subcutaneous into affected dermatome } \\
\text { or }\end{array}$ \\
\hline Spinal Stenosis & $\begin{array}{c}\text { Various spinal vertebrae, } \\
\text { cervical or lumbar }\end{array}$ & $\begin{array}{c}\text { NSAIDs, Muscle relaxants, Tricyclic } \\
\text { antidepressants, opioids, anticonvulsants, } \\
\text { Epidural steroid injection, surgery }\end{array}$ & Intrathecal or intraganglionic routes \\
\hline Arachnoiditis & Lumbar spinal cord & $\begin{array}{c}\text { NSAIDs, Muscle relaxants, Tricyclic } \\
\text { antidepressants, opioids, anticonvulsants, } \\
\text { steroids, TENS, Spinal cord stimulation }\end{array}$ & Intrathecal or intraganglionic routes \\
\hline
\end{tabular}

guided techniques are available [80, 81]. In addition to trigeminal or post-herpetic neuralgia, the intraganglionic approach may be very useful for certain cancers, like pancreatic cancer, that are localized to one or two dermatomes. This approach becomes especially important when the pain is located in the upper thoracic or cervical dermatomes, where the intrathecal route is too difficult to use. Precise injection is vital in these areas as loss of noxious thermal sensation in the face and hands can cause multiple ADL problems. It may also be possible to treat other ganglia. Frequently the celiac plexus is blocked by injection of neuroablative agents like alcohol. RTX could replace these less selective chemoablative procedures while using the same types of image-guided needle placement methods [81]. These data suggest that intraganglionic RTX infusion may provide a new treatment for a variety of pain syndromes in which unilateral effects are needed and perineural or peripheral subcutaneous treatments are not feasible.

Table 4 outlines several non-malignant chronic pain conditions that might be treated with intraganglionic or intrathecal RTX. A factor to consider is that RTX would only be give once. Some of the patient populations are relatively young when the pain problem occurs and the single injection may represent a more effective alternative to conventional analgesic treatments such as opioids. It is also worth considering the idea of giving a low dose of RTX and removing only some of the TRPV1-expressing fibers. This may convert a debilitating pain syndrome to a more manageable problem yet retain some inflammatory pain sensation. Again, the treatment can be tailored to the particular pain situation and RTX needs to be injected only once.

\section{RTX: Intrathecal Administration}

Unlike the intraganglionic, skin and nerve injections, which are well circumscribed by anatomical factors, the intrathecal route of RTX administration can be used to treat large areas of the body with only one injection. When given into the lumbar cistern, the drug can access the entire cauda equina, which encompasses much of the lumbar and sacralcoccygeal afferents. Depending on the exact parameters of the intrathecal injection procedure, the volume administered, and the dose, the drug can spread even higher. Given this arrangement, pain originating from most of the lower half of the body can be effectively treated with an intrathecal RTX injection. Similar to the intraganglionic route of administration, the intrathecal route also produces an irreversible effect. The drug accesses the neuronal cell bodies in the DRG and to their axons in the dorsal roots. Once the cell body or axons are compromised by RTXinduced calcium cytotoxicity, they may be permanently ablated. Thus, the use of RTX by this route has to be considered carefully, especially in cases of non-malignant pain.

\section{Rat and Dog Studies}

Preclinical studies of intrathecally administered RTX in rats demonstrate a loss of peptidergic primary afferents in the dorsal spinal cord, loss of TRPV1 neurons in the DRG and behavioral effects consistent with the loss of neurons that sense noxious heat and inflammatory hyperalgesia [41, 47, reviewed in 82]. The effect was long lasting, selective and, similar to the other routes, produced analgesia, but did not affect motor activity, coordination or mechanosensitivity. The analgesic actions of RTX were extended to cancer pain by treatment of dogs with naturally occurring osteosarcoma [37]. Canine osteosarcoma is similar to human bone cancer and usually affects the long bones in a limb. The dogs were enrolled into the study because of pain that was unresponsive to conventional management with NSAIDS, opioids and steroids. Prior to RTX administration, the animals would not bear weight on the limb with the osteosarcoma. RTX was injected into either the lumbar cistern (for hind limb tumors) or the cisterna magna (for forelimb tumors). Because of the acute pain provoked at the time of RTX administration, the injection was performed under general anesthesia with endotracheal intubation [37]. Intrathecal RTX induced a transient hypertensive and tachycardic response with an onset at $5 \mathrm{~min}$ and then these parameters returned to control by $60 \mathrm{~min}$ without any medical intervention. These hemodynamic changes occurred in both control animals and in those with osteosarcoma.

Recovery was generally uneventful and blood and urine specimens collected before and 2 weeks after RTX injection showed no significant alterations. Pain intensity was evaluated by the owners with a visual analog scale (VAS) at 2, 6, 10 and 14 weeks after RTX administration. The average VAS rating pretreatment was 53.0 on a $100-\mathrm{mm}$ scale. PostRTX, the VAS rating dropped to 8.0 by week 2 and the animals became ambulatory, walking on four legs. The VAS 
ratings remained at this low level until week 14 when the formal observation period ended. In addition, it was possible to reduce or eliminate other analgesic drug treatments in the majority of the animals. The longest post-injection survival was 9 months and strong pain control was still evident. Importantly, at no time was a change in 'personality' noted for any of the dogs, suggesting that higher CNS functions were unaffected by RTX, and no bladder or bowel dysfunction was reported [37].

\section{Human Clinical Trial}

The positive results in rat pain models and canine cancer pain led to a Phase I clinical trial with intrathecal RTX administration in human cancer pain patients [83]. To date, six patients have been treated. Much of what was seen in the canine study has, so far, translated into the human study. However, unlike the canine osteosarcoma, in which the tumor presentation was similar, albeit in different limbs, the human cases were more complex. No two patients had exactly the same tumor presentation or constellation of pain problems, even if the origin of the cancer was the same (e.g. cervical cancer). Nonetheless, all patients experienced substantial analgesia with no significant adverse effects. The study continues to recruit patients.

In the human study RTX was given by intrathecal injection into the lumbar cistern, consequently, eligibility criteria were for patients with pain from the mid-chest down. We did not include patients with, for example, pain from head and neck cancer because to achieve an effect in the cervical cord with a lumbar injection would produce loss of TRPV1 afferents throughout the entire body. Also we wanted to retain thermal sensitivity in the hands and face in order for the patients to sample their thermal environment and reduce burn risk due to lack of feedback. For cancer pain in the upper half of the body other routes are possible. Injection into the cisterna magna would expose afferents of the spinal trigeminal nucleus and cervical cord to the RTX solution. This would likely cause loss of thermal sensation in the face and extending down to the arms and hands. In fact, with cisternal administration in the initial canine doseranging study we observed loss of forepaw thermal sensation [37]. Intraganglionic administration might offer a more selective approach when cancer pain involves the trigeminal or cervical regions.

If all goes well, the use of RTX for treating cancer pain would be a new addition to the pharmacological management of pain. The fact that RTX only has to be given once and that other analgesic drugs may be reduced or discontinued could greatly improve a patient's quality of life. RTX treatment would be especially important in cases where opiates are failing, where high doses of opioids are needed to control pain at the expense of patient consciousness, and in cases where non-specific neuroablative procedures were being considered for palliation. In these situations the benefit of using RTX to the patient's quality of life can be substantial and raises the question of when to intervene? This may become even more pertinent if the cancer can be arrested but not necessarily eliminated yet a severe pain problem is present. There are many additional questions that can be addressed in subsequent studies. Among them are how to optimize and/or customize administration for individual cases and how to develop administration procedures that do not require general anesthesia? Lastly, if it is safe and effective, how can this treatment be made available to all those who need it?

\section{RTX: Systemic Administration and Mechanical Allodynia}

Compared to some of the other routes, systemic administration of RTX is less well studied. RTX is a potent irritant [84] and the $\mathrm{LD}_{50}$ for RTX by oral administration is $\sim 150 \quad \mathrm{mg} / \mathrm{kg} \quad$ (http://www.lookchem.com/resiniferatoxin/). The systemic route probably has little therapeutic value for treating human pain problems, however, animal studies analyzing this route have raised several important questions. In mice, the intraperitoneal route has been used not as an analgesic manipulation, but rather as mechanism to induce mechanical allodynia. Studies of allodynia were first conducted in 2008 by Hsieh et al. [45], in which a small diameter nerve fibre sensory neuropathy was generated through a single systemic (i.p.) injection of $50 \mu \mathrm{g} / \mathrm{kg}$ RTX. The aim was to demonstrate the potential therapeutic effects of 4-methylcatechol (4MC) to promote regeneration of unmyelinated nerves. Further testing showed that systemic RTX produced mechanical allodynia as detected by a decrease in threshold of paw withdrawal in a von Frey hair test. This occurred in mice [45] and rats [85]. The rat model of RTX-induced mechanical allodynia was used to demonstrate anti-allodynic efficacy of pulsed radiofrequency (PRF) administration in the early stages of this neuropathy model [85]. In mice with neuropathy, the systemic RTXinduced mechanical hypersensitivity produced an increase in expression of $\mathrm{P} 2 \mathrm{X} 3$ receptors within skin nerves. In these mice it was demonstrated that intraplantar injection of P2X3 antagonists relieved the mechanical allodynia in a dosedependent manner, suggesting that $\mathrm{P} 2 \mathrm{X} 3$ receptor antagonists might be therapeutic for denervation-related neuropathic pain problems.

The relationship between mechanical allodynia, nerve injury and i.p. RTX was also examined in another study of mechanical allodynia induced by loose ligatures placed on the sciatic nerve in rats [86]. Rats with tactile allodynia and thermal hyperalgesia displayed a reduction of thermal hyperalgesia after systemic RTX but the mechanical allodynia was not affected, nor was mechanical allodynia induced. Additionally treatment of multiple lumbar ganglia by RTX also did not induce mechanical allodynia. Rather, RTX gave a clear antiallodynic effect. These discordant results suggest that the induction of mechanical allodynia by RTX may be susceptible to a procedural variable, although, as expected, all of the studies demonstrated a loss of thermal pain sensation.

\section{Innervation and Allodynia}

Despite differences, these studies raise the idea that "too much" may not be beneficial. It is possible that, in some studies, the systemic administration of RTX removed all of the TRPV1-expressing afferents from dorsal horn second order neurons and that this produced a synaptic rearrangement that resulted in allodynia to mechanical modalities of stimulation. It is well known that nociceptive CGRP-containing primary afferents have collaterals that spread up and down the spinal cord dorsal horn over multiple 
Table 5. Chronic pain conditions that are spatially diffuse or lack of distinct localization that may not be appropriate for RTX treatment

\begin{tabular}{|c|c|c|}
\hline Condition & Location & Qualifications \\
\hline Fibromyalgia & Disseminated & $\begin{array}{l}\text { Pain may be too diffuse for local injection unless a primary trigger point } \\
\text { can be identified }\end{array}$ \\
\hline Headache & Head & Requires a clear site of origin for a local injection \\
\hline Sickle Cell Disease & $\begin{array}{l}\text { General Vascular } \\
\text { Involvement }\end{array}$ & Pain is likely too distributed for local injection \\
\hline Myofascial Pain & Various locations & $\begin{array}{l}\text { Pain may not be sufficiently localized for an injection unless a primary } \\
\text { trigger point can be identified }\end{array}$ \\
\hline Abdominal pain & No distinct site for needle placement & $\begin{array}{l}\text { Conditions in which there is a definable trigger zone (e.g. as seen on } \\
\text { endoscopy) may be amenable to a local injection }\end{array}$ \\
\hline $\begin{array}{l}\text { Central Pain (Post-Stroke, Multiple } \\
\text { Sclerosis related pain) }\end{array}$ & Diffusely located & $\begin{array}{c}\text { Central pain often extends over large areas of the body like the whole left } \\
\text { or right side, or the lower half of the body. }\end{array}$ \\
\hline
\end{tabular}

segments [87-89]. Incomplete lesions still leave considerable amounts of CGRP remaining in nerves [87, 88], which is measurable by radioimmunoassay [89]. An incomplete effect, with residual collaterals above and below the zone of RTX effect supplying synapses, likely prevents synaptic rearrangement. On the other hand, a very thorough removal of TRPV1 nerve endings over large segments of the spinal cord may leave the dendrites of second order neurons open for colonization by new, nearby synaptic inputs. This is known to happen in hippocampus where denervation of cholinergic fibers from the septum causes sympathetic fibers from nearby blood vessels to sprout into the dentate gyrus $[90,91]$. Excessive synaptic stripping may be possible with intrathecal RTX administration, but the spread of the drug would have to be large to overcome the overlap of ascending and descending afferent collaterals. In this regard we did not observe an induction of mechanical allodynia, even with doses of RTX up to 2000 nanograms given intrathecally to rats. This was enough to produce loss of capsaicin eye wipe even though the drug was administered by lumbar puncture (Iadarola and Keller, unpublished). These data suggest that, with clinically useful routes and doses, RTX is not prone to inducing denervation-dependent side effects.

\section{SUMMARY}

\section{RTX as an Interventional Approach to Personalized Pain Medicine}

The various sites for RTX administration: peripheral nerve terminals in skin or joints, injection around or into a peripheral nerve, injection directly into the trigeminal or dorsal root ganglion, and finally injection into the CSF around the spinal cord (intrathecal) constitute progressively greater levels of intervention for pain control. This is a useful operational framework in terms of developing procedures and studying the underlying neurobiology. Using anatomical and neurological principles, RTX intervention can be personalized to the patient's particular pain problem. The capacity to adapt the treatment to the requirements of the pain problem is a unique feature of the TRPV1 agonist approach.
In addition to localized injections for incisional pain, certain neuropathic pain patients with a definable trigger zone may be ideal candidates for subcutaneous RTX injection. For example, Gracely, Lynch and Bennett [92] reported the following case:

"A 52-year-old woman developed severe shooting pains in the elbow following ulnar nerve transposition surgery in 1988. This spontaneous pain was accompanied by mechanoallodynia at a site of unusual hair growth distal to the elbow. The patient was evaluated during 2 local anesthetic blocks of the hyperpigmented region near the surgical scar at the elbow; this was the site that evoked severe radiating pain when palpated. Infiltration of $5 \mathrm{ml}$ of $1.5 \%$ lidocaine in the hyperpigmented region resulted in complete anesthesia at the site of injection after $2 \mathrm{~min}$. Three minutes after infiltration all spontaneous pain was absent. Testing by hair movement, blowing on the skin, cotton wisp and von Frey filaments (3.6 g) showed that allodynia had disappeared completely in the forearm while touch sensitivity was preserved."

It is reasonable to speculate that in this type of patient, localized RTX treatment might provide long-term pain relief since it would inactivate the nerve endings in the trigger zone. Additional chronic pain conditions in which peripheral, localized application of RTX could provide therapeutic benefit are listed in Table $\mathbf{3}$.

Other conditions may benefit from a broader investigation of routes of administration. Post herpetic neuralgia $(\mathrm{PHN})$ provides an interesting condition for the various routes by which RTX can be delivered. The Qutenza capsaicin patch provides relief from PHN pain, indicating that pain can be controlled by inactivating the nerve endings in the skin. This supports the use of RTX by localized infiltration into the skin. At the same time, if the area of affected skin is large, perineural or direct intra-ganglionic application might provide more efficient interventional approaches. Clearly, the intrathecal route is not appropriate for such a localized problem. For cancer, the intrathecal route may be the most appropriate but even here, depending on the specific presentation, treatment by nerve or ganglionic injection may be more appropriate. 
Each route has its own set of advantages and constraints, but what pain problems are likely not appropriate for the TRPV1 agonist approach? Table $\mathbf{5}$ gives several examples. The common feature is a lack of clear localization of the "peripheral generator" [92] and obviously post-stroke central pain problems.

This review was both retrospective and prospective. It is meant to show not only the potential of RTX for pain treatment, but even more generally, the potential of the TRPV1 agonist approach. RTX is very potent and specific and there seem to be very few negative side effects consequent to its use. Thus, we hope it can enter into widespread clinical application. However, as a vanilloid agonist RTX causes pain upon administration and with further investigation it may be possible to generate other TRPV1 agents with more favorable pharmacological characteristics $[93,94]$.

\section{CONFLICT OF INTEREST}

The authors confirm that this article content has no conflicts of interest.

\section{ACKNOWLEDGEMENTS}

This research was supported but the Division of Intramural Research of the National Institute of Dental and Craniofacial Research.

\section{REFERENCES}

[1] Huang SC, Korlipara VL. Neurokinin-1 receptor antagonists: a comprehensive patent survey. Expert Opin Ther Pat 2010; 20(8): 1019-45.

[2] Stewart JM. Bradykinin antagonists: discovery and development. Peptides 2004; 25(3): 527-32.

[3] Cannon GW, Breedveld FC efficacy of cyclooxygenase-2-specific inhibitors, Am J Med 2001; 110(Suppl 3A): 6S-12S.

[4] Walpole CS, Bevan S, Bovermann G, et al. The discovery of capsazepine, the first competitive antagonist of the sensory neuron excitants capsaicin and resiniferatoxin. J Med Chem 1994; 37(13): 1942-54.

[5] Patel L, Lindley C. Aprepitant--a novel NK1-receptor antagonist. Expert Opin Pharmacother 2003; 4(12): 2279-96.

[6] Burnstock G. Do some nerve cells release more than one transmitter? Neuroscience 1976; 1(4): 239-48.

[7] Hökfelt T, Arvidsson U, Cullheim S, et al. Multiple messengers in descending serotonin neurons: localization and functional implications. J Chem Neuroanat 2000; 18(1-2): 75-86.

[8] Hnasko TS, Edwards RH. Neurotransmitter corelease: mechanism and physiological role. Annu Rev Physiol 2012; 74: 225-43.

[9] Battaglia G, Rustioni A. Coexistence of glutamate and substance $P$ in dorsal root ganglion neurons of the rat and monkey. J Comp Neurol 1988; 277(2): 302-12.

[10] Mishra SK, Holzman S, Hoon MA. A nociceptive signaling role for neuromedin B J Neurosci 2012; 32(25): 8686-95.

[11] Benyamin R, Trescot AM, Datta S, et al. Opioid complications and side effects. Pain Physician 2008; 11(2 Suppl): S105-20.

[12] Caterina MJ, Schumacher MA, Tominaga M, Rosen TA, Levine JD, Julius D. The capsaicin receptor: a heat-activated ion channel in the pain pathway. Nature 1997; 389: 816-24.

[13] Jordt SE, McKemy DD, Julius D. Lessons from peppers and peppermint: the molecular logic of thermosensation. Curr Opin Neurobiol 2003; 13(4): 487-92.

[14] Tominaga M, Caterina MJ. Thermosensation and pain. J Neurobiol 2004; 61(1): 3-12.

[15] Moran MM, McAlexander MA, Bíró T, Szallasi A. Transient receptor potential channels as therapeutic targets. Nat Rev Drug Discov 2011; 10(8): 601-20.

[16] Hardie RC. TRP channels and lipids: from Drosophila to mammalian physiology. J Physiol 2007; 578(Pt 1): 9-24.
[17] Montell C. The history of TRP channels, a commentary and reflection. Pflugers Arch 2011; 461(5): 499-506.

[18] Kort ME, Kym PR. TRPV1 antagonists: clinical setbacks and prospects for future development. Prog Med Chem 2012; 51: 5770 .

[19] Waxman SG, Cummins TR, Dib-Hajj S, Fjell J, Black JA. Sodium channels, excitability of primary sensory neurons, and the molecular basis of pain. Muscle Nerve 1999; 22(9): 1177-87.

[20] Akopian AN, Sivilotti L, Wood JN. A tetrodotoxin-resistant voltage-gated sodium channel expressed by sensory neurons. Nature 1996; 379(6562): 257-62.

[21] Lane NE, Schnitzer TJ, Birbara CA, Mokhtarani, et al. Tanezumab for the treatment of pain from osteoarthritis of the knee. N Engl J Med 2010; 363(16): 1521-31.

[22] Dubovy P. Wallerian degeneration and peripheral nerve conditions for both axonal regeneration and neuropathic pain induction. Ann Anat 2011; 193: 267-75.

[23] Taveggia C, Feltri ML, Wrabetz L. Signals to promote myelin formation and repair. Nat Rev Neurol 2010; 6(5): 276-87.

[24] Ma Q. Labeled lines meet and talk: population coding of somatic sensations. J Clin Invest 2010; 120(11): 3773-8.

[25] Brown MT, Murphy FT, Radin DM, Davignon I, Smith MD, West CR. Tanezumab Reduces Osteoarthritic Knee Pain: results of a Tandomized, Double-Blind, Placebo-Controlled Phase III Trial. J Pain 2012; 13(8): 790-8.

[26] Garber K. Fate of novel painkiller mAbs hangs in balance. Nat Biotechnol 2011; 29 (3): 173-4.

[27] Rowbotham MC, Nothaft W, Duan WR, et al. Oral and cutaneous thermosensory profile of selective TRPV1 inhibition by ABT-102 in a randomized healthy volunteer trial. Pain 2011; 152(5): 1192200.

[28] Gavva NR, Treanor JJ, Garami A, et al. Pharmacological blockade of the vanilloid receptor TRPV1 elicits marked hyperthermia in humans. Pain 2008; 136(1-2): 202-10.

[29] Wong GY, Gavva NR. Therapeutic potential of vanilloid receptor TRPV1 agonists and antagonists as analgesics: recent advances and setbacks. Brain Res Rev 2009; 60(1): 267-77.

[30] Mannes AJ, Iadarola MJ. Complete pain relief: potential problems and diagnostic solutions. Nat Clin Pract Neurol 2007; 3(12): 648-9.

[31] Mannes A, Iadarola M. Potential downsides of perfect pain relief. Nature 2007; 446 (7131): 24.

[32] Vlassakov KV, Narang S, Kissin I. Local anesthetic blockade of peripheral nerves for treatment of neuralgias: systematic analysis. Anesth Analg 2011; 112(6): 1487-93.

[33] McGee HT, Fraunfelder FW. Toxicities of topical ophthalmic anesthetics. Expert Opin Drug Saf 2007; 6: 637-40.

[34] Bennett GJ. What is spontaneous pain and who has it? J Pain 2012; 13(10): 921-9.

[35] Neubert JK, Karai L, Jun JH, Kim HS, Olah Z, Iadarola MJ. Peripherally induced resiniferatoxin analgesia. Pain 2003; 104(12): 219-28.

[36] Kárai L, Brown DC, Mannes AJ, et al. Deletion of vanilloid receptor 1-expressing primary afferent neurons for pain control. J Clin Invest 2004; 113(9):1344-52.

[37] Brown DC, Iadarola MJ, Perkowski SZ, et al. Physiologic and antinociceptive effects of intrathecal resiniferatoxin in a canine bone cancer model. Anesthesiology 2005; 103(5): 1052-9.

[38] Bates BD, Mitchell K, Keller JM, et al. Prolonged analgesic response of cornea to topical resiniferatoxin, a potent TRPV1 agonist. Pain 2010; 149(3): 522-8.

[39] Kissin I, Bright CA, Bradley EL Jr. Selective and long-lasting neural blockade with resiniferatoxin prevents inflammatory pain hypersensitivity. Anesth Analg 2002; 94(5): 1253-8.

[40] Kissin I, Freitas CF, Mulhern HL, DeGirolami U. Sciatic nerve block with resiniferatoxin: an electron microscopic study of unmyelinated fibers in the rat. Anesth Analg 2007; 105(3): 825-31.

[41] Olah Z, Szabo T, Karai L, et al. Ligand-induced dynamic membrane changes and cell deletion conferred by vanilloid receptor 1. J Biol Chem 2001; 276(14): 11021-30.

[42] Olah Z, Karai L, Iadarola MJ. Anandamide activates vanilloid receptor 1 (VR1) at acidic $\mathrm{pH}$ in dorsal root ganglia neurons and cells ectopically expressing VR1. J Biol Chem 2001; 276(33): 31163-70.

[43] Gover TD, Moreira TH, Kao JP, Weinreich D. Calcium regulation in individual peripheral sensory nerve terminals of the rat. J Physiol 2007; 578(Pt 2): 481-90. 
[44] Gover TD, Moreira TH, Weinreich D. Role of calcium in regulating primary sensory neuronal excitability. Handb Exp Pharmacol 2009; (194): 563-87.

[45] Hsieh YL, Chiang H, Tseng TJ, Hsieh ST. Enhancement of cutaneous nerve regeneration by 4-methylcatechol in resiniferatoxin-induced neuropathy. J Neuropathol Exp Neurol 2008; 67(2): 93-104.

[46] Hsieh YL, Chiang H, Lue JH, Hsieh ST. P2X3-mediated peripheral sensitization of neuropathic pain in resiniferatoxin-induced neuropathy. Exp Neurol 2012; 235(1): 316-25.

[47] Bishnoi M, Bosgraaf CA, Premkumar LS. Preservation of acute pain and efferent functions following intrathecal resiniferatoxininduced analgesia in rats. J Pain 2011; 12(9): 991-1003.

[48] Mitchell K, Bates BD, Keller JM, et al. Ablation of rat TRPV1expressing Adelta/C-fibers with resiniferatoxin: analysis of withdrawal behaviors, recovery of function and molecular correlates. Mol Pain 2010; 6: 94.

[49] Yang HY, Mitchell K, Keller JM, Iadarola MJ. Peripheral inflammation increases Scya2 expression in sensory ganglia and cytokine and endothelial related gene expression in inflamed tissue. J Neurochem 2007; 103(4): 1628-43.

[50] Wallace M, Pappagallo M. Qutenza ${ }^{\circledR}$ : a capsaicin $8 \%$ patch for the management of postherpetic neuralgia. Expert Rev Neurother 2011; 11(1): 15-27.

[51] Simone DA, Nolano $M$, Johnson $T$, Wendelschafer-Crabb G, Kennedy WR. Intradermal injection of capsaicin in humans produces degeneration and subsequent reinnervation of epidermal nerve fibers: correlation with sensory function. J Neurosci 1998; 18(21): 8947-59.

[52] Iadarola MJ, Douglass J, Civelli O, Naranjo JR. Increased spinal cord dynorphin mRNA during peripheral inflammation. NIDA Res Monogr 1986; 75: 406-9.

[53] Iadarola MJ, Douglass J, Civelli O, Naranjo JR. Differential activation of spinal cord dynorphin and enkephalin neurons during hyperalgesia: evidence using cDNA hybridization. Brain Res 1988; 455(2): 205-12.

[54] Hollins M. Somesthetic senses. Annu Rev Psychol 2010; 61: 24371.

[55] Lopes C, Liu Z, Xu Y, Ma Q. Tlx3 and runx1 act in combination to coordinate the development of a cohort of nociceptors, thermoceptors, and pruriceptors. J Neurosci 2012; 32(28): 9706-15.

[56] Mishra SK, Tisel SM, Orestes P, Bhangoo SK, Hoon MA. TRPV1lineage neurons are required for thermal sensation. EMBO J 2011; 30(3): 582-93

[57] Marmigère F, Ernfors P. Specification and connectivity of neuronal subtypes in the sensory lineage. Nat Rev Neurosci 2007; 8(2): 11427.

[58] Li L, Rutlin M, Abraira VE, Cassidy C, et al. The functional organization of cutaneous low-threshold mechanosensory neurons. Cell 2011; 147(7): 1615-27.

[59] Liu Y, Ma Q. Generation of somatic sensory neuron diversity and implications on sensory coding. Curr Opin Neurobiol 2011; 21(1): 52-60.

[60] Franck MC, Stenqvist A, Li L, et al. Essential role of Ret for defining non-peptidergic nociceptor phenotypes and functions in the adult mouse. Eur J Neurosci 2011;33(8): 1385-400.

[61] Zylka MJ, Rice FL, Anderson DJ. Topographically distinct epidermal nociceptive circuits revealed by axonal tracers targeted to Mrgprd. Neuron 2005; 45(1): 17-25.

[62] Ivanavicius SP, Blake DR, Chessell IP, Mapp PI. Isolectin B4 binding neurons are not present in the rat knee joint. Neuroscience 2004; 128(3): 555-60.

[63] Cantillon M, Vause E, Sykes D, Tagoe M. Safety, tolerability and efficacy of intraoperative ALGRX 4975 in a randomized, doubleblind, placebo-controlled study of subjects undergoing bunionectomy. J Pain 2005; 6(3): S48.

[64] Aasvang EK, Hansen JB, Malmstrøm J, et al. The effect of wound instillation of a novel purified capsaicin formulation on postherniotomy pain: a double-blind, randomized, placebocontrolled study. Anesth Analg 2008; 107(1): 282-91.

[65] Kárai LJ, Russell JT, Iadarola MJ, Oláh Z. Vanilloid receptor 1 regulates multiple calcium compartments and contributes to $\mathrm{Ca} 2+-$ induced $\mathrm{Ca} 2+$ release in sensory neurons. J Biol Chem 2004; 279(16): 16377-87.
[66] Cantillon M, Vause E, Sykes D, Russell R, Moon A, Hughes S. Preliminary safety, tolerability and efficacy of ALGRX 4975 in osteoarthritis (OA) of the knee. J Pain 2005; 6(3): Suppl S39.

[67] Apostolidis A, Gonzales GE, Fowler CJ. Effect of intravesical Resiniferatoxin (RTX) on lower urinary tract symptoms, urodynamic parameters, and quality of life of patients with urodynamic increased bladder sensation. Eur Urol 2006; 50(6): 1299-305.

[68] Lazzeri M, Beneforti P, Spinelli M, Zanollo A, Barbagli G, Turini D. Intravesical resiniferatoxin for the treatment of hypersensitive disorder: a randomized placebo controlled study. J Urol 2000; 164(3 Pt 1): 676-9.

[69] Chen TY, Corcos J, Camel M, Ponsot Y, Tu le M. Prospective, randomized, double-blind study of safety and tolerability of intravesical resiniferatoxin (RTX) in interstitial cystitis (IC). Int Urogynecol J Pelvic Floor Dysfunct 2005; 16(4): 293-7.

[70] Payne CK, Mosbaugh PG, Forrest JB, et al. ICOS RTX Study Group (Resiniferatoxin Treatment for Interstitial Cystitis). Intravesical resiniferatoxin for the treatment of interstitial cystitis: a randomized, double-blind, placebo controlled trial. J Urol 2005; 173(5): 1590-4.

[71] Brown DL, Fink BR. The history of neural blockade and pain management. In: Cousins MJ, Bridenbaugh PO, Eds. Neural blockade in clinical anesthesia and management of pain, 3rd ed. Philadelphia: Lippincott-Raven 1998; pp 3-34.

[72] Kissin I. Vanilloid-induced conduction analgesia: selective, dosedependent, long-lasting, with a low level of potential neurotoxicity. Anesth Analg 2008; 107(1): 271-81.

[73] Acs G, Palkovits M, Blumberg P.M. Comparison of $[3 \mathrm{H}]$ resiniferatoxin binding by the vanilloid(capsaicin) receptor in dorsal root ganglia, spinal cord, dorsal vagal complex, sciatic and vagal nerve and urinary bladder of the rat. Life Sci 1994; 55: 101726.

[74] Guo A, Vulchanova L, Wang J, Li X, Elde R. Immunocytochemical localization of the vanilloid receptor 1 (VR1), relationship to neuropeptides, the $\mathrm{P} 2 \mathrm{X} 3$ purinoceptor and IB4 binding sites. Eur J Neurosci 1999; 11: 946-58.

[75] Caudle RM, Karai L, Mena N, et al. Resiniferatoxin-induced loss of plasma membrane in vanilloid receptor expressing cells. Neurotoxicology 2003; 24(6): 895-908.

[76] Gover TD, Kao JP, Weinreich D. Calcium signaling in single peripheral sensory nerve terminals. J Neurosci 2003; 23(12): 47937.

[77] Tender GC, Walbridge S, Olah Z, et al. Selective ablation of nociceptive neurons for elimination of hyperalgesia and neurogenic inflammation. J Neurosurg 2005; 102(3): 522-5.

[78] Neubert JK, Mannes A, Laszlo K, et al. Perineural resiniferatoxin selectively inhibits inflammatory hyperalgesia. Mol Pain 2008; 4: 3.

[79] Kissin I, Freitas CF, Bradley EL Jr. Perineural resiniferatoxin prevents the development of hyperalgesia produced by loose ligation of the sciatic nerve in rats. Anesth Analg 2007; 104(5): 1210-6.

[80] Brull R, Perlas A, Chan VW. Ultrasound-guided peripheral nerve blockade. Curr Pain Headache Rep 2007; 11(1): 25-32.

[81] Peng PW, Tumber PS. Ultrasound-guided interventional procedures for patients with chronic pelvic pain - a description of techniques and review of literature. Pain Physician 2008; 11(2): 215-24.

[82] Iadarola MJ, Mannes AJ. The vanilloid agonist resiniferatoxin for interventional-based pain control. Curr Top Med Chem 2011; 11(17): 2171-9.

[83] Mannes M, Hughes M, Quezado Z, et al. A potent TRPV1 agonist: Intrathecal administration to treat severe pain associated with advanced cancer — case report. J Pain 2010; 11(4): S43.

[84] Evans AT, Gordge PC, Sahni V, Evans FJ. The potent irritancy of the daphnane orthoester, resiniferatoxin, exhibits features of a mixed aetiology. J Pharm Pharmacol 1992; 44(4): 361-3.

[85] Tanaka N, Yamaga M, Tateyama S, Uno T, Tsuneyoshi I, Takasaki M. The effect of pulsed radiofrequency current on mechanical allodynia induced with resiniferatoxin in rats. Anesth Analg 2010; 111(3): 784-90.

[86] Ossipov MH, Bian D, Malan TP Jr, Lai J, Porreca F. 10. Lack of involvement of capsaicin-sensitive primary afferents in nerveligation injury induced tactile allodynia in rats. Pain 1999; 79(2-3): 127-33. 
[87] Traub RJ, Allen B, Humphrey E, Ruda MA. Analysis of calcitonin gene-related peptide-like immunoreactivity in the cat dorsal spinal cord and dorsal root ganglia provide evidence for a multisegmental projection of nociceptive C-fiber primary afferents. J Comp Neurol 1990; 302(3): 562-74.

[88] Traub RJ, Solodkin A, Ruda MA. Calcitonin gene-related peptide immunoreactivity in the cat lumbosacral spinal cord and the effects of multiple dorsal rhizotomies. J Comp Neurol 1989; 287(2): 22537.

[89] Traub RJ, Iadarola MJ, Ruda MA. Effect of multiple dorsal rhizotomies on calcitonin gene-related peptide-like immunoreactivity in the lumbosacral dorsal spinal cord of the cat: a radioimmunoassay analysis. Peptides 1989; 10(5): 979-83.

[90] Crutcher KA, Brothers L, Davis JN. Sympathetic noradrenergic sprouting in response to central cholinergic denervation; a histochemical study of neuronal sprouting in the rat hippocampal formation. Brain Res 1981; 210(1-2): 115-28.
[91] Madison R, Davis JN. Sprouting of noradrenergic fibers in hippocampus after medial septal lesions: contributions of the central and peripheral nervous systems. Exp Neurol 1983; 80(1): 167-77.

[92] Gracely RH, Lynch SA, Bennett GJ. Painful neuropathy: altered central processing maintained dynamically by peripheral input. Pain 1992; 51(2): 175-94.

[93] Roh EJ, Keller JM, Olah Z, Iadarola MJ, Jacobson KA. Structureactivity relationships of 1,4-dihydropyridines that act as enhancers of the vanilloid receptor 1 (TRPV1). Bioorg Med Chem 2008; 16(20): 9349-58.

[94] Kaszas K, Keller JM, Coddou C, et al. Small molecule positive allosteric modulation of TRPV1 activation by vanilloids and acidic pH. J Pharmacol Exp Ther 2012; 340(1): 152-60.

(C) Iadarola and Gonnella; Licensee Bentham Open.

This is an open access article licensed under the terms of the Creative Commons Attribution Non-Commercial License (http://creativecommons.org/licenses/by-nc/3.0/) which permits unrestricted, non-commercial use, distribution and reproduction in any medium, provided the work is properly cited. 